Kommentar zum Beitrag von Jacques de Haller «Medizinischer Nachwuchs: Wollen wir Diplomierte oder Ärzte?» [1]

\title{
Wollen wir nicht lieber diplomierte Ärzte? Sind fachliche und soziale Kompetenzen Gegensätze?
}

\section{Klaus-Dieter Hänsgen}

Direktor Zentrum für Testentwicklung und Diagnostik
Universität Freiburg

\begin{abstract}
Die Zulassungsbegrenzung zum Medizinstudium (Numerus clausus oder NC) und die Auswahl Geeigneter sind unterschiedliche Fragen, die einfach zusammenhängen: Wenn ein NC notwendig ist, muss eine Auswahl erfolgen. Ersteres hängt praktisch nur von den zur Verfügung stehenden Ausbildungskapazitäten ab. Das ggf. notwendige Zulassungsverfahren zum Medizinstudium in der Schweiz wurde und wird laufend optimiert. Es müssen dabei aber die richtigen Diagnosen gestellt und erst dann ggf. geeignete Therapien angewendet werden. Ein implizierter Widerspruch zwischen fachlichen Fähigkeiten und sozialen Kompetenzen, zwischen Studien- und Berufseignung ist so nicht haltbar.
\end{abstract}

\section{«Lässt sich das Fehlen dieser Fähigkeiten wirklich mit sozialen Kompetenzen ausgleichen?»}

Mehr Information mi Quellen unter: www.ztd.ch (Der WIKI-Blog zu EMS und seinen Randbedingungen).

1 De Haller J. Medizinischer Nachwuchs: Wollen wi Diplomierte oder Ärzte? Schweiz Ärztezeitung. 2014;95(4):129-32.

\section{Korrespondenz:}

Prof. Dr. Klaus-D. Hänsgen ZTD Universität Freiburg Rte d'Englisberg 9 CH-1763 Granges-Paccot haensgen[at]bluewin.ch
Es scheint Einigkeit darüber zu bestehen, dass man die Zulassung zum Medizinstudium begrenzen muss (nur dies nennt man Numerus clausus, NC). Gegenüber 2006 haben die Universitäten mit NC in Humanmedizin 2014 ihre Kapazitäten bereits um 38\% erhöht - die Nachfrage ist trotzdem stärker gewachsen, sodass nur noch rund $1 / 3$ der Personen einen Platz erhält. Diese Nachfrage «explodiert» nicht nur in der Schweiz. Ob die Arztserien, die durch Ärztemangel-Diskussion implizierte Zukunftssicherheit des Berufes, der soziale Status, das Einkommen oder eine vermeintliche Vereinfachung der Anforderungen in Studium Ursachen sind, bleibt offen. Selbst wenn man die Kapazitäten weiter ausbaut, kann man in den Universitäten mit NC objektiv nicht mehr alle zulassen, ohne Qualität einzubüssen, Reformen zurückzunehmen oder das erste bis zweite Jahr als «Massenstudium» mit allen Nachteilen zu gestalten - weil man nicht nur «Diplomierte» will. Die erst durch die Zugangsbegrenzung ermöglichten Reformen (frühe Patientenorientierung, Kleingruppen u. a.) erfordern ausreichende Betreuungskapazität und dienen dazu, neben reinem «Faktenpauken» die Persönlichkeitsentwicklung von Beginn an zu fördern.

Die richtige Auswahl, um das «beschränkte Gut» Studienplätze optimal zu nutzen, ist wichtig. Wenn man am Ende auch mehr Ärzte und nicht mehr Studienabbrüche will, müssen so viele wie möglich das Studium abschliessen und dann auch im Arztberuf arbeiten. So lange das Medizinstudium anspruchsvoll hinsichtlich der mathematisch-naturwissenschaftlichen
Fähigkeiten bleibt, müssen diese ausreichend vorhanden sein. Kann man unter diesen Bedingungen auf eine Selektion aufgrund der Studienerfolgsprognose verzichten? Lässt sich das Fehlen dieser Fähigkeiten wirklich mit sozialen Kompetenzen ausgleichen? «Menschlich nett, aber leider durchgefallen»? Es gibt sogar laute Forderungen seitens der Universitäten, den Naturwissenschaftsaspekt als Eignungskriterium stärker zu gewichten, weil der Wissensstand im Mittel abgenommen habe, bzw. zu heterogen sei.

Ein Gegensatz von Studien- und Berufserfolg ist nach unserer Literaturkenntnis nirgends empirisch nachgewiesen worden - Studienerfolg ist eher eine Facette des Berufserfolges, weil kognitive Fähigkeiten auch für die spätere ärztliche Berufstätigkeit, für die Anforderungen lebenslangen Lernens, bedeutend sind. Hier scheint eher das Klischee vorzuliegen, dass kognitiv fähige Leute «Fachidioten» seien - dies ist aber wirklich nur ein Klischee ohne empirische Absicherung.

Wenn heute Ärzte «gestresster» sind, weniger Zeit für die Behandlung des einzelnen Falles haben bzw. aus ökonomischen Gründen keine Hausbesuche mehr machen, die Anforderungen durch die Komplexität der vielen neuen diagnostischen und therapeutischen Möglichkeiten grösser werden und der Arzt objektiv nicht mehr der «liebevolle Onkel oder die liebevolle Tante Doktor» aus unserer Erinnerung sein kann, muss man die Bewältigbarkeit der beruflichen Anforderungen durch den Arzt verbessern - und dies nicht auf Kosten der Freizeit. Denn auch prosoziales Verhalten kann und muss man lernen und dafür förderliche Bedingungen im Studium und Beruf schaffen.

Wenn man fachbezogene soziale Kompetenzen bei der Auswahl einbeziehen will, so liegen diese zum Zeitpunkt vor Studienbeginn ebenso wenig ausgeformt vor wie das Fachwissen. Schon das Verwenden «fachnaher» Anforderungen beim Prüfen (z.B. PseudoPatientengespräche) ist eigentlich ein Kunstfehler das richtige Verhalten soll ja erst im Studium erlernt werden. Was man prüfen kann, sind Voraussetzungen zum sozialen Lernen, wo sich Menschen auch unterscheiden. In Israel (und auch anderswo) ist das Verfahren zweistufig: Alle müssen zuerst einen Test absolvieren, und man prüft nur einen Teil der Personen differenzierter - anders kann man dies nicht bezahlen. Es wurde übrigens auch in der Schweiz bei Einführung des NC überlegt, ob man ergebnisabhängig zu Interviews einlädt - hohe Kosten und unklarer Nutzen liessen davon Abstand nehmen. 
Der erwähnte sogenannte «Psychometric Entrance Test» (PET) des NITE in Israel ist mit dem Schweizer Eignungstest für das Medizinstudium (EMS) absolut vergleichbar, erfasst ebenso «Fähigkeiten, die für akademische Studien erforderlich sind» [2] (verbal reasoning, quantitative reasoning, English). Die ersten beiden Faktoren sind nahezu identisch abgedeckt. Sprachkenntnisse müssen wir in der Schweiz nicht testen.

Der Schweizer EMS ist ein psychometrischer Test objektiv, zuverlässig und valide, was die wichtigsten Gütekriterien für solche Tests sind. Ein Unterschied zum PET ist, dass im EMS die Aufgaben weniger trai-

\section{«Fachspezifische Studierfähigkeitstests weisen neben Maturanoten die höchste Prognosegüte auf.»}

2 www.nite.org.il/index.php/ en/tests/psychometric/ psychometric-test-format. html

3 Siehe www.ztd.ch/w/index. php?title=Matura

4 www.unifr.ch/ztd/ems/agab. pdf

5 Dazu z.B. Situational Judgement Test: www.unifr.ch/ ztd/ems/doc/SJT.pdf

6 Zur Heterogenität siehe WIKIPEDIA: https://de. wikipedia.org/wiki/ Soziale_Kompetenz

7 www.unifr.ch/ztd/ self-assessment/ nierbar sind. Spätestens seit der Metaanalyse zur Erfolgsvorhersage von Schuler und Mitarbeitern [3] wissen wir, dass fachspezifische Studierfähigkeitstests neben Maturanoten die höchste Prognosegüte aufweisen - diese Werte erreicht der EMS nachweislich. Also besser als reine Wissens- oder allgemeine Studierfähigkeitstests, viel besser als Interviews.

Falls mit «Psychometrietests» Wissenstests gemeint wären: Die haben neben einer grösseren Trainierbarkeit einen weiteren wesentlichen Nachteil: Sie messen sinnbildlich nur, wie viel «Fett» jemand angesetzt hat (und fragen nicht nach dem Aufwand, also der Effizienz). Studierfähigkeitstests messen sinnbildlich, ob man ein guter «Futterverwerter» (Lerner, sich neues Wissen effektiv anzueignen) ist. Genau dies ist für ein erfolgreiches Studium gefragt.

Die «Gretchenfrage»: Wenn man etwas testet/ prüft/misst und dadurch Personen auswählt, muss man sicher sein, dass sich damit der gewünschte Erfolg (sei dies Studienerfolg, Berufserfolg oder «guter Arzt sein») zuverlässig vorhersagen lässt und die Auswahl damit auch gerecht ist. Andernfalls kann man auch losen oder würfeln und hat dann wieder mehr Studienabbrüche.

Was ist ein guter Arzt, und welche Rolle spielen soziale Kompetenzen dabei? Zum einen gibt es in der Literatur dazu wenige Analysen - viele bedienen ebenfalls eher Klischees. Böswillig könnte man z. B. sagen: Wer wirklich sozial kompetent ist, wird so lange nicht Hausarzt, wie objektiv die Bedingungen dort schlechter sind als in anderen Fächern. Verlangt man von Studienanwärtern ein Bekenntnis zum Hausarzt als Berufsziel, kann dies leicht ein Lippenbekenntnis werden, auch die vermeintlich «objektiveren» Fragen sind verfälschbar, und das Wunsch-Verhalten ist gut trainierbar. Assessment Center in der Wirtschaft - dann mit fachnahen Aufgaben, wo also die Ausbildung dafür schon vorausgesetzt werden kann - setzen auf deutlich längere Durchführungszeiten, um etwas «hinter die Fassade» schauen zu können.

«Ethisches Verhalten», «Dienstbereitschaft» oder die Fähigkeit, Hilfe anzufordern, sind Dimensionen, in denen soziale Erwünschtheit eine grosse Rolle spielt. Intelligente Personen können sich in der Regel auch besser «verstellen» als andere. Unsere Befragung nach dem EMS zeigt, dass Personen mit hohen Testleistungen noch deutlich offener sind für die Disziplinwahl als Personen mit tieferen Testleistungen (die primär eine Spezialarzt-/Facharztkarriere einschlagen wollen) [4]. Auch hier zeigt sich nicht, dass weniger fähige Kandidaten vermehrt Hausarzt werden möchten.

Dass soziale Kompetenzen für den Arztberuf wichtig sind, findet breite Zustimmung. Ersten Streit gibt es zwischen Fachleuten, was soziale Kompetenzen genau sind - praktisch alle nichtintellektuellen Merkmale werden irgendwo dazugerechnet. «Gibt es das gleiche Anforderungsprofil für alle Fachgebiete der Medizin, und wo ist dort die Schnittmenge?», ist die nächste umstrittene Frage. Der Streit wird heftiger, wenn es um Messverfahren geht, die verfälschungssicher, wenig trainierbar und vor allem valide im Sinne der Vorhersage zukünftiger Entwicklungen sein müssen. Insofern haben wir es beim EMS nicht «aufgegeben», soziale Kompetenzen bzw. genauer die Fähigkeit zum Erwerb derselben zu messen, sondern suchen weiter nach einem wissenschaftlich fundierten wie bezahlbaren Verfahren $[5,6]$. Self Assessments sind vertretbar, um schon heute bei der Studienwahl zu sensibilisieren [7].

Die Berücksichtigung sozialer Kompetenzen kann entweder unmittelbar im EMS oder in einem zweistufigen Verfahren wie z. B. in Israel geschehen. Was immer man dort aber testet/prüft/beurteilt: Es muss ebenso nachgewiesen werden, dass ein gewünschtes Zielverhalten dadurch wissenschaftlich ausreichend genau vorhergesagt werden kann. Hier besteht bei allen uns bekannten Modellen solcher «Kurzzeit-Stationsbetriebe» grosser Nachholbedarf. Konsistenz reicht dabei nicht und heisst nur, dass mehrere Aufgaben das Gleiche messen oder mehrere Beurteiler etwas gleich einschätzen. Was ich damit genau messe, weiss ich trotzdem noch nicht. Der sogenannten «Eindrucksvalidität» («Das wirkt sozial kompetent, das wird schon bedeutsam sein») darf man dabei nicht aufsitzen.

\section{Fazit}

So lange nicht alle interessierten Personen zugelassen werden können, haben wir mit dem EMS ein valides psychometrisch fundiertes Verfahren, was den Studienerfolg und damit die Wahrscheinlichkeit des Abschlusses nachweislich vorhersagt.

Klagen über fehlende soziale Kompetenzen der Beteiligten im Gesundheitswesen können viele Ursachen haben - man sollte die objektiven Berufsanforderungen nicht vergessen und muss auch kompetenzförderliche Studienbedingungen schaffen.

Alle Beteiligten am EMS sind offen für mehr «Soft Skills», wie z.B. soziale Kompetenzen - im EMS oder auch in zweistufigen Verfahren. Guten Gewissens kann man keines der heute «am Markt» verfügbaren Verfahren als so ausreichend validiert betrachten, dass man ohne Glauben auskommt, wenn man damit über «Schicksale» von Menschen entscheiden will. 